
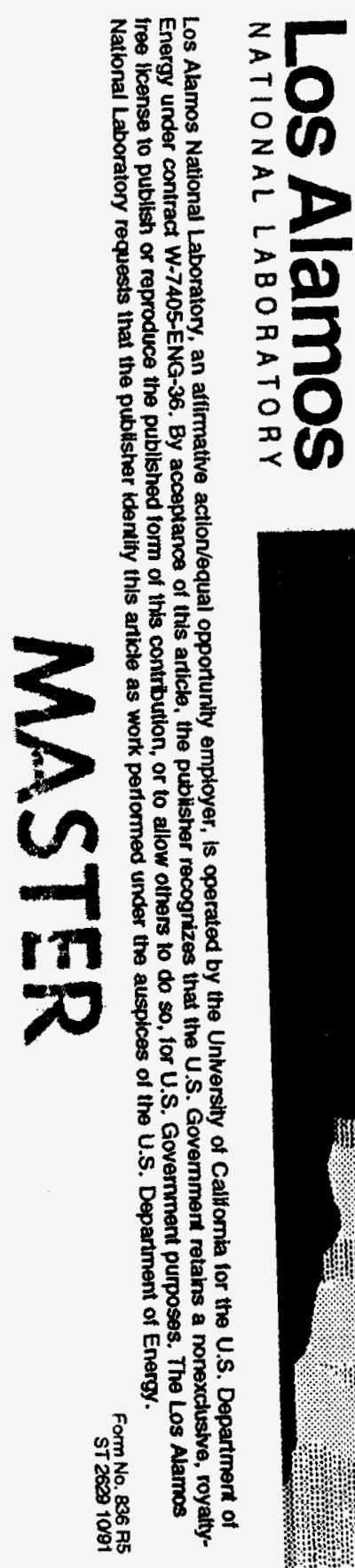

This report was prepared as an account of work sponsored by an agency of the United States Government. Neither the United States Government nor any agency thereof, nor any of their employees, makes any warranty, express or implied, or assumes any legal liability or responsibility for the accuracy, completeness, or usefulness of any information, apparatus, product, or process disclosed, or represents that its use would not infringe privately owned rights. Reference herein to any specific commercial product, process, or service by trade name, trademark manufacturer, or otherwise does not necessarily constitute or imply its endorsement recommendation, or favoring by the United States Government or any agency thereof. The vien and opinions of authors expressed herein do not necessarily state or reflect those of the United States Government or any agency thereof.

总

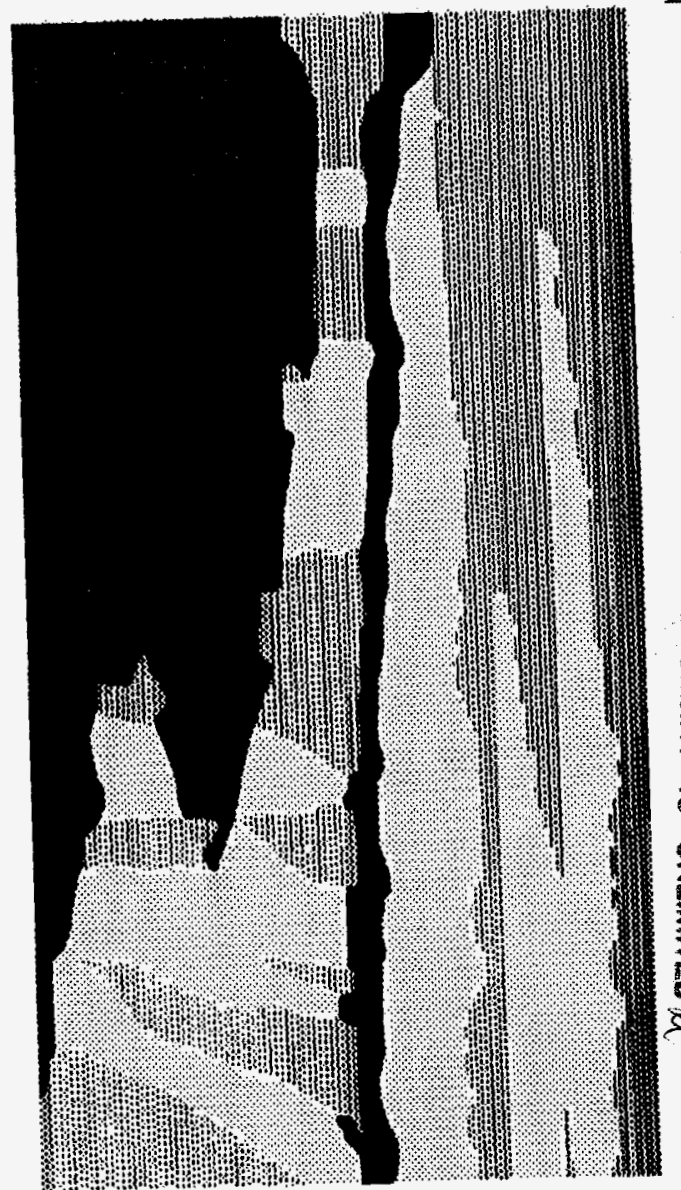

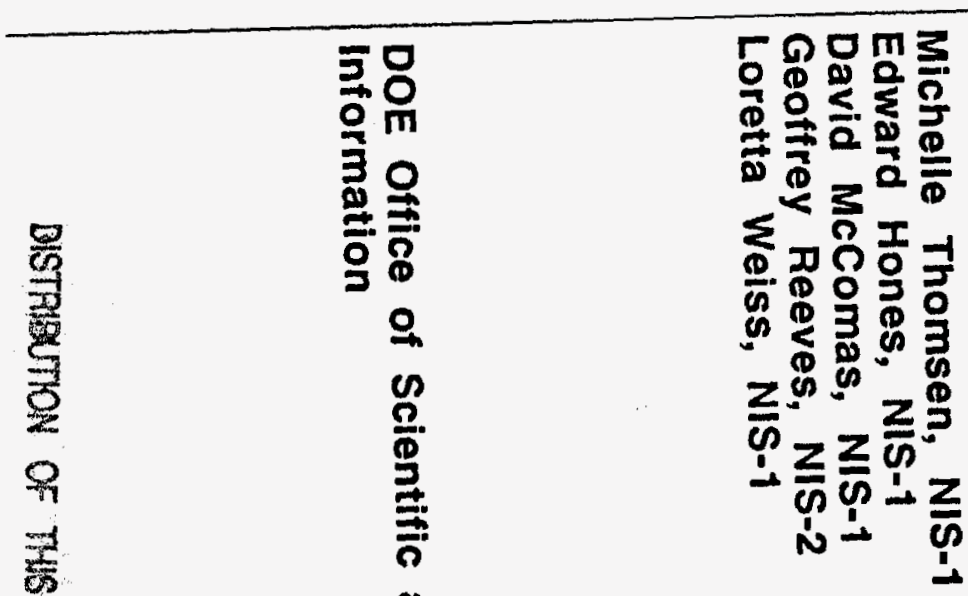

อ

ㅇ

몽

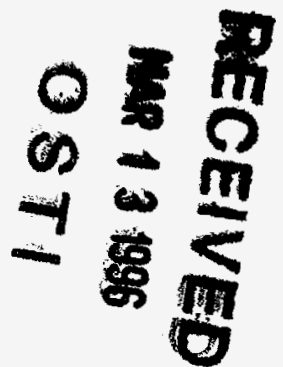




\section{DISCLAIMER}

Portions of this document may be illegible in electronic image products. Images are produced from the best available original document. 


\title{
Measuring the Magnetic Connectivity of the Geosynchronous Region of the Magnetosphere
}

Michelle Thomsen*, Edward Hones, David McComas, Geoffrey Reeves, and Loretta Weiss

\begin{abstract}
This is the final report of a three-year, Laboratory-Directed Research and Development (LDRD) project at the Los Alamos National Laboratory (LANL). The purpose of this project was to determine the magnetic connectivity of the geosynchronous region of the magnetosphere to the auroral zone in the polar ionosphere in order to test and refine current magnetospheric magnetic field models. We used plasma data from LANL instruments on three geosynchronous satellites and from USAF instruments on three low-altitude, polar-orbiting, DMSP satellites. Magnetic connectivity is tested by comparing plasma energy spectra at DMSP and geosynchronous satellites when they are in near conjunction. The times of closest conjugacy (i.e., best spectral match) are used to evaluate the field models. We developed the tools for each step of the process and applied them to the study of a one-week test set of conjunctions. We automated the analysis tools and applied them to four months of twosatellite observations. This produced a database of about 130 definitive magnetic conjunctions. We compared this database with the predictions of the widely-used Tsyganenko magnetic field model and showed that in most cases one of the various parameterizations of the model could reproduce the observed field line connection. Further, we explored various measurables (e.g., magnetospheric activity indices or the geosynchronous field orientation) that might point to the appropriate parameterization of the model for these conjunctions, and ultimately, for arbitrary times.
\end{abstract}

\section{Background and Research Objectives}

One of the most urgent problems of magnetospheric research is that of magnetic field line mapping between the low-altitude ionosphere and atmosphere and the high-altitude

\footnotetext{
*Principal investigator, e-mail: mthomsen@lanl.gov
} 
magnetosphere. This is a central problem of magnetospheric physics because the magnetic field is the path by which high-altitude processes driven by the solar wind (the magnetized plasma flowing outward from the solar corona) are coupled down to low altitudes to produce terrestrial effects like the aurorae. Recent spacecraft observations and empirical magnetospheric field models have called into question the previously accepted paradigm of connection between the aurora and the magnetosphere, implying significant revision of current hypotheses regarding the source and acceleration of the precipitating magnetospheric particles responsible for producing the aurora. New observational insights are urgently needed to help clarify this mapping and to establish more definitively the overall magnetic topology of the magnetosphere and its response to varying solar wind conditions.

Our objective is to test and improve currently available magnetospheric field models by making a large number of two-point observations of plasma distribution functions -- one set of measurements at geosynchronous distance near the magnetospheric equator and the other 800 $\mathrm{km}$ above the polar regions of Earth -- and, through comparisons of the energy spectra, to identify locations of closest magnetic conjugacy. The observations use plasma analyzers on three geosynchronous satellites and three polar orbiting DMSP satellites. Each of the many observed pairs of magnetically conjugate points is compared with predictions of conjugacy made by the best current field models, and systematic deviations are identified to point the way to improvements in the models.

\section{Importance to LANL's Science and Technology Base and National R\&D Needs}

The space physics capabilities and expertise that exist at Los Alamos are essential ingredients for participation in arms control programs and in space-based non-proliferation efforts. This LDRD project utilizes, exercises, and expands these capabilities and, at the same time produces results that should have important impacts on activities of the national and international magnetospheric physics community. Magnetospheric physics is a leading effort in space physics today. This fact is manifested in the International Solar Terrestrial Physics (ISTP) program, which has now begun and will continue through the 1990's. The program comprises coodinated launches and operations of a number of satellites developed by the National Aeronautics and Space Administration (NASA), the European Space Agency (ESA), the Japanese Institute of Space and Astronautical Science (ISAS), and Russia. Data from the LANL instruments on synchronous satellites are also playing an important role in the ISTP effort. To effectively analyze and interpret data from these various sources will require magnetic field models and mapping capability superior to any that are presently available. Our 
study will contribute materially to the definition of such improved models, which will ultimately form the foundation of a national space weather capability.

\section{Scientific Approach and Results}

Plasma measurements from three geosynchronous satellites and three DMSP satellites are compared for intervals when the satellites are estimated to have been in approximate magnetic conjunction. The conjunction estimates are made using the present best magnetospheric model. The three synchronous satellites are situated at different local times, each circling the Earth once per day. The three DMSP satellites are in three different $800 \mathrm{~km}$ polar orbits, the plane of the DMSP-8 orbit being the 06-18 hour local time plane, DMSP-9 being in the 10-22 hour plane and DMSP-10 being in the 08-20 hour plane.

The first requirement for a magnetic conjunction is that a synchronous satellite be near the magnetic local time plane of one of the DMSP satellites. The second is that the DMSP satellite traverse field lines that reach out to the radial distance of the synchronous satellite. The latter can happen twice each orbit -- once in the northern hemisphere and once in the southern hemisphere. Orbit calculations, together with the magnetic field model, allow us to make approximate predictions of times of close conjunctions. At such times we compare the ion and electron spectra obtained by DMSP each second with a spectrum measured at synchronous orbit at the approximate time (within a few minutes) of the predicted conjunction.

There may be substantial pitch-angle dependence of the synchronous orbit spectra and so it is necessary that the synchronous orbit measurement be made as near to the atmospheric loss cone (i.e., as nearly along the field line direction) as possible. There is not a magnetometer on the synchronous satellites but the field line direction can be determined from the angular distribution of energetic electrons. The plasma spectral measurements taken at $6 \mathrm{x}$ $24=144$ look directions then allow us to choose measurements made within a few degrees of that field direction. Those spectra are then compared with the second-by-second measurements from DMSP until a best correlation is found, identifying the moment of closest conjugacy.

In the first year of the project we acquired a week's worth of digital electron and proton data from each of the polar-orbiting satellites DMSP-8, -9 , and -10 and identified 78 times within that week when there was close azimuthal conjugacy between one of the DMSP satellites and one of the geosynchronous satellites (1989-046 and 1990-095). We processed the geosynchronous electron and proton data for all 33 of the conjunctions between 1989-046 and a DMSP satellite, as well as for 4 of the 1990-095 conjunctions. We co-plotted the DMSP and geosynchronous electron spectra for all of these 37 events and did a preliminary assessment of the times of best spectral agreement for about half of those. For one of those 
cases we did the full mapping from high to low altitude based on the best currently available magnetospheric field model, the so-called Tsyganenko- 89 model. The model is parametrized by magnetic activity level, and we performed the mapping for two different activity levels: $\mathrm{Kp}=4$ (close to the true value at the time of the conjunction) and $\mathrm{Kp}=0$. For this case we found a good magnetic mapping between the low-altitude DMSP satellite and the geosynchronous satellite when the $K p=4$ field was used, but not when the $K p=0$ field was used.

In the second year of the project we finished our assessment of the times of best spectral agreement for the 37 events we had previously processed for the week of March 7-12, 1991. For each of the 20 of these events for which there was good agreement between two independent observers on the times of good spectral match, we performed the magnetic mapping using the Tsyganenko-89 field model parametrized by $\mathrm{Kp}=0,1,2,3,4,5$. We found that in almost all of the cases there was a value of $\mathrm{Kp}$ for which the Tsyganenko model gave an acceptable mapping between the satellites. However, the particular value of $\mathrm{Kp}$ which gave good mapping was typically not the same as the actual measured value of $\mathrm{Kp}$ during the threehour interval encompassing the conjunction. We conclude from this preliminary study that the model probably contains the appropriate elements to produce a correct mapping, but that the measured geophysical $\mathrm{Kp}$ is not a very good index for selecting the value to assign to the parameter "Kp" in the model. These results were presented at the Fall meeting of the American Geophysical Union and at the International Solar Terrestrial Physics Symposium in Sendai, Japan. They also form the basis of a paper submitted to the Journal of Geophysical Research [1]. We also used our one-week test set of conjunctions to begin our comparative assessment of different magnetic field models, beyond the Tsyganenko model we had previously explored. These results were presented at the 1994 COSPAR meeting in Hamburg, Germany.

In the third and final year of the project, we finished automating our tools and streamlining the analysis process in order to undertake the systematic analysis of large numbers of events. Using four months of DMSP data (one month from each equinox and solstice season), we applied this automated technique to create a database of approximately 130 definitive conjunction events that allows us to investigate the dependence of magnetic connectivity on geomagnetic activity, dipole tilt, and magnetic local time. We also continued our comparison of different magnetic field models beyond the Tsyganenko model. In addition, we explored other measured geophysical parameters (such as Dst, AE, or the equatorward edge of the diffuse aurorae) to see if they provide a better pointer to the appropriate value of " $\mathrm{Kp}$ " to use in the Tsyganenko field model. The various results of this work were presented at the Fall and Spring meetings of the American Geophysical Union and at the 21st General Assembly of the International Union of Geophysics and Geodesy. The search for a suitable pointer to the appropriate parameterization of the Tsyganenko model is described in a paper submitted to 
J. Geophys. Res. [2], and a paper summarizing the definitive mappings is in preparation [3]. Based on preliminary results of our completed LDRD project, we have obtained NASA funding to continue pursuing various aspects of this work. Finally, the database we have generated will continue to be a valuable tool for testing and refining candidate magnetospheric field models.

\section{References}

[1] Hones, E. W., M. F. Thomsen, G. D. Reeves, L. A. Weiss, D. J. McComas, and P. T. Newell, "Observational Determination of Magnetic Connectivity of the Geosynchronous Region of the Magnetosphere to the Auroral Oval," J. Geophys. Res., vol. 101, p. 2629 (1995).

[2] Weiss, L. A., M. F. Thomsen, G. D. Reeves, and D. J. McComas, "An Examination of the Tsyganenko (T89a) Field Model Using a Database of Two-Satellite Magnetic Conjunctions," J. Geophys. Res., submitted (1996).

[3] Weiss, L. A., M. F. Thomsen, G. D. Reeves, and D. J. McComas, "The Ionospheric Footprint of Geosynchronous Satellites," Geophys. Res. Lett., to be submitted (1996). 